Cubero et al., J.Am.Chem.Soc. 2003

\title{
THEORETICAL STUDY OF A NEW DNA STRUCTURE: THE ANTIPARALLEL HOOGSTEEN DUPLEX
}

\author{
Elena Cubero $^{1}$, Nicola G. A. Abrescia ${ }^{2}$, Juan A. Subirana ${ }^{3}$, F.Javier Luque ${ }^{4 *}$ \\ and Modesto Orozco ${ }^{1,5 *}$
}

\begin{abstract}
The structure of a new form of the duplex DNA, the antiparallel Hoogsteen duplex, is studied in polyd(AT) sequences by means of state-of-the-art molecular dynamics simulations in aqueous solution. The structure, which was found to be stable in all the simulations, has many similarities with the standard Watson-Crick duplex in terms of general structure, flexibility and molecular recognition patterns. Accurate MM-PB/SA (and MM-GB/SA) analysis show that the new structure has an effective energy similar to that of the B-type duplex, while it is slightly disfavoured by intramolecular entropic considerations. Overall, MD simulations strongly suggest that the antiparallel Hoogsteen duplex is an accessible structure for a polyd(AT) sequence, which might compete under proper experimental conditions with normal B-DNA. MD simulations also suggest that chimeras containing Watson-Crick duplex and Hoogsteen antiparallel helices might coexist in a common structure, but preserving the differential characteristics of both type of structures.
\end{abstract}

\footnotetext{
${ }^{1}$ Molecular Modeling \& Bioinformatic Unit. Institut de Recerca Biomèdica. Parc Científic de Barcelona. Josep Samitier 1-5. Barcelona 08028. Spain

${ }^{2}$ Division of Structural Biology. Wellcome Trust Centre for Human Genetics. Oxford University. Oxford OX3 7BN. United Kingdom

3 Departament d'Enginyeria Química. Universitat Politécnica de Catalunya. Avgda Diagonal 647. Barcelona 08028. Spain

${ }^{4}$ Departament de Fisicoquímica. Facultat de Farmacia. Universitat de Barcelona. Avgda Diagonal 643. Barcelona 08028. Spain

${ }^{5}$ Departament de Bioquímica i Biologia Molecular. Facultat de Química. Universitat de Barcelona. Martí i Franquès 1. Barcelona 08028. Spain
} 
Cubero et al., J.Am.Chem.Soc. 2003

\section{INTRODUCTION}

Under physiological conditions the DNA is mostly found as a right-handed double helix that shows a conformation, named B-DNA, not far from that suggested by Watson and Crick (1). However, fifty years after the discovery of the double helix, it is clear that the DNA is very polymorphic, and that its equilibrium conformation can change depending on sequence and environment (2-4). Repetitive sequences are especially rich in terms of accessible helical structures (for reviews see refs. 2, 4 and 7). For example, poly(GC), poly $(\mathrm{G})$ and poly(GA) sequences are known to form easily left-handed Z-DNA (5), fourstranded helical structures (6), and triple helices (7), respectively. Other examples are sequences containing poly $(\mathrm{C})$ or poly $(\mathrm{CT})$ tracks, which can generate the i-form of DNA $(8,9)$, and sequences rich in polyd(A) tracks, which can yield parallel duplexes showing reverse Watson-Crick pairings, parallel duplexes with Hoogsteen pairings (10), or parallel duplexes involving d(A.A) ${ }^{+}$dimers (2). The poly(AT) sequences are probably those exhibiting the widest range of accessible structures (11-21). Thus, crystal structures of $\mathrm{d}(\mathrm{AT})_{2}$ show a distorted B-type conformation $(11,12)$. Other crystal structures even show a larger distortion, suggesting the existence of a very special type of B DNA named TADNA (13). Early fiber diffraction data on polyd(AT) sequences suggested left-handed models (14), but this has not been confirmed by higher resolution techniques. On the other hand, polyd(AT) fibers generated under low hydration conditions show a D form of DNA (15), while at low temperature the C-form is obtained (16). Very recently, Subirana and coworkers (17) have reported the high resolution structure of the d(ATATAT) duplex, which corresponds to a new structural motif of DNA consisting of an antiparallel righthanded double helix with adenosines in the syn conformation and unexpected Hoogsteen $d(A \cdot T)$ pairings. To our knowledge, the existence of this form in aqueous solution has not been confirmed by NMR experiments.

The possibility to have different H-bond patterns for the A-T pair (see Figure 1) is known since the sixties (22). Accurate theoretical calculations (23) showed that in fact the Watson-Crick pairing is not the best recognition mode for the A.T pair, and that all the four recognition patterns shown in Figure 1 are accessible, at least in the gas phase. Molecular 
dynamics simulations have also shown that stable helices can be built for both duplexes (10a) and triplexes (24) using Hoogsteen A-T pairings. Experimentally the Hoogsteen recognition mode is found in different structures of DNA and RNA (25), including the parallel triplexes $(7,26)$, where it stabilizes both $\mathrm{d}(\mathrm{A}-\mathrm{T} \cdot \mathrm{T})$ and $\mathrm{d}(\mathrm{G}-\mathrm{C} \cdot \mathrm{C})$ triads. Very interestingly, Hoogsteen pairs are common in complexes between duplex DNA and drugs or proteins $(18,27-29)$. In conjunction with the biological role of $\mathrm{d}(\mathrm{AT})_{\mathrm{n}}$ regions $(27,30)$, these findings strongly suggest an important role for Hoogsteen pairings in the modulation of gene expression $(18,27)$. In summary, recent theoretical and experimental information points out that the apparently exotic Hoogsteen interaction might be common and have a large biological significance.

In this paper we present a wide and systematic molecular dynamics (MD) study of the poly(AT) duplex in aqueous solution at low ionic strength. Both the normal WatsonCrick (B-form) and antiparallel Hoogsteen (apH) structures (17) are studied using large MD trajectories for 4, 6, 8, 10,12,14 and 16-mer duplexes (more than $40 \mathrm{~ns}$ of trajectories are presented here). The structure, dynamics and recognition properties of the apH helix in water are determined and compared with those of the standard B-DNA duplexes. The relative stability of apH and B-type duplexes is discussed using information derived from the MD simulations. Finally, trajectories of chimeras containing fragments of apH and BDNAs are generated to analyse the impact of Hoogsteen pairings in the structure of a long canonical piece of DNA. The results obtained here complement previous X-Ray data by Abrescia et al. (17) and provide a complete picture on a new and intriguing structure of the DNA duplex.

\section{METHODS}

In order to analyse with good statistical accuracy (10a) the structure and stability of apH and B duplexes for poly(AT) sequences, we built starting structures for complementary duplexes $\mathrm{d}(\mathrm{AT})_{\mathrm{n} / 2}$ for $\mathrm{n}=4,6,8,10,12,14$ and 16 using standard Arnott's parameters for B-DNA (31) and the crystal structure data for the apH-DNA (17). The fourteen different structures were neutralized by adding a suitable number of $\mathrm{Na}^{+}$ions 
(10a), and immersed in rectangular boxes containing between 1000 and 3500 water molecules. All the systems were optimized, thermalized (298K) and equilibrated using our standard multistage protocol $(24,32)$. The equilibrated structures were then subjected to 2 ns (4, 6, 8, 14 and 16-mer) and $5 \mathrm{~ns}$ (10 and 12-mer) of MD simulation at constant temperature $(298 \mathrm{~K})$ and pressure $(1 \mathrm{~atm})$ with standard relaxation times of $0.2 \mathrm{ps}$. Periodic boundary conditions were used to simulate a diluted environment and the Particle Mesh Ewald method was used to account for long-range electrostatic effects (33). Ewald tolerance of $5 \times 10^{-6}$ was used in conjunction with a grid spacing of $1 \AA$ and a four order interpolation scheme. SHAKE (34) was used to constrain all the bonds at their equilibrium positions, which allowed us to use a $2 \mathrm{fs}$ time step for integration of Newton equations. AMBER-98 (35) and TIP3P (36) force-fields were used to describe DNA and water. All the MD simulations were performed using the AMBER6.0 suite of programs (37).

The MD simulations of apH and $\mathrm{B}$ duplexes were analysed to evaluate their relative stability. For this purpose, the free energy of every structure was computed as shown in eq. 1 (38). MD averages $(<>)$ for each contribution in eq. 1 were obtained after dropping the first nanosecond of every trajectory. $E_{\text {intra }}$ was computed using the standard AMBER-98 force-field (35). The solvation contribution $\left(\mathrm{G}_{\mathrm{sol}}\right)$ was determined using two different approaches (for a discussion of the two techniques see ref. 39): i) finite difference PoissonBoltzman (PB) calculations as implemented in the MEAD program (40) for initial and final grid spacings of 1 and $0.4 \AA$, and ii) the Generalized-Born (GB) method (41) as implemented in AMBER6.0. A dielectric constant of 80 was used to represent water, while dielectric constants of 1 or 2 were used to represent the solute. Finally, the contribution to the free energy of the system due to the intramolecular DNA entropy was determined by using Schliter's method (42) as described by Harris et al (43). We should note that test calculations using Andricioae \& Karplus method (44) did not lead to any significant difference with respect to Schliter-type calculations, and were then not included. To avoid artifacts in the averaging of the different terms the end-base pairs were removed from the analysis.

$$
G \approx\left\langle E_{\text {int } r a}\right\rangle+\left\langle G_{\text {sol }}\right\rangle-T \Delta S_{\text {int } r a}=\left\langle E_{\text {effec }}\right\rangle-T S_{\text {int } r a}
$$


Calculations noted in eq. 1 were performed for the different apH and B-type structures. Taking advantage of the linear relationship between the length of the oligonucleotide and the free energy (see ref. 10a and below), we can then derive estimates of the relative stability of apH and B structures with very good statistical accuracy (see Results).

Description of the interaction properties of apH (and B) structures was obtained from cMIP calculations for an $\mathrm{O}^{+}$probe molecule $(24,32,44)$. Solvation around the structures was represented by integrating the water population along the trajectory as explained elsewhere $(24,32)$. The analysis of molecular flexibility was performed using principal component analysis (PCA) method as described by Sherer et al (46). Principal components were obtained by diagonalization of the covariance matrix obtained after recentering all the collected snapshots. The result of this procedure is a set of eigenvalues and the corresponding eigenvectors. Eigenvalues can be manipulated to obtain vibrational frequencies and provide useful information on the magnitude of the flexibility of DNA along their essential movements. The eigenvectors projected into the Cartesian space illustrate the nature of the essential movements of the DNA molecule. As shown previously (47), we can compare the similarity between two unitary eigenvectors using the dot product, obtaining then a quantitative measure of the similarity between two essential movements. The strategy can be expanded (see eq. 2 and reference 47 ) to quantitatively compute the similarity between a reduced set of eigenvectors, which are known to explain more than a certain amount of variance in the trajectory (47). Note that due to the orthogonality between principal components, two identical trajectories will yield a similarity index $\gamma$ of 1 , while due to the limited number of eigenvectors considered two unrelated trajectories will have $\gamma$ equal to 0 .

$$
\gamma_{A B}=\frac{1}{n} \sum_{j=1}^{n} \sum_{i=1}^{n}\left(v_{i}^{A} \bullet v_{j}^{B}\right)^{2}
$$


where $\mathrm{A}$ and $\mathrm{B}$ stand for two different trajectories of equal length (for the same or different structures), $v$ stands for an unit eigenvector and $\mathrm{n}$ is taken as 10 , a number of modes which accounts for around $80 \%$ of the variance for the 10 and 12-mer oligonucleotides considered here. To reduce errors, only backbone atoms (up to $\mathrm{Cl}^{\prime}$ ') were considered in comparison between $\mathrm{B}$ and apH helices.

A relative similarity index ( $\kappa)$, which accounts for the uncertainties intrinsic to any time-limited MD trajectory, can be obtained by normalizing the absolute index $\gamma_{\mathrm{AB}}$ by the self-similarities obtained in the $A$ and $B$ trajectories, $\gamma_{A A}{ }^{T}$ and $\gamma_{B B}{ }^{T}$ (see eq. 3). Selfsimilarities are easily obtained by comparing the first and last halves of the same trajectory. Both $\gamma$ and $\kappa$ are 1 for two identical trajectories and 0 when they are orthogonal. As noted in reference 47, this index runs also from 1 (identical) to 0 (unrelated), and has the advantage to be less simulation-sensitive than absolute similarity index $(\gamma)$

$$
\kappa_{A B}=2 \frac{\gamma_{A B}}{\left(\gamma_{A A}^{T}+\gamma_{B B}^{T}\right)}
$$

Geometrical analysis was performed using the PTRAJ module of the in AMBER6.0 program, as well as with in-house programs. Helical analysis was carried out using Olson's X3DNA program (48) and Curves5.3 (49). Base pairs at the ends of the duplex were removed in all the cases. All calculations were performed at the Centre de Supercomputació de Catalunya (CESCA) and in workstations in our laboratories.

\section{RESULTS AND DISCUSSION}

\section{Stability of trajectories of polyd(AT) $)_{n}$ in B and apH forms.}

All the trajectories of B and apH structures show stable helical conformations (see Figure 2), as noted in the small root-mean square deviations (RMSds) with respect to the MD-averaged structures (see Table 1). In general, lower RMSds are found for the apH 
duplexes than for the B-like ones, suggesting that the former sample a narrower region of the configurational space, probably due to a greater rigidity of the apH structure (see below). Comparison of 2 and $5 \mathrm{~ns}$ trajectories demonstrates that the trajectories converge well in the 2 ns time scale, and that apparently no relevant transitions are expected for longer simulation times. The all-atom RMSd of the apH trajectories with respect to the crystal structure (PDB entry 1gqu.pdb; (17)) are small ( $<2 \AA$ in all the cases), showing that MD simulations in aqueous solution sample regions of the configurational space very close to the crystal structure. This agreement supports the suitability of the MD simulation to study the apH duplex, and the ability of X-ray data to represent a reasonable conformation of the polyd(AT $)_{\mathrm{n}}$ duplex in dilute solution.

The B-like trajectories are closer to the B than to the A form, but the RMSds with respect to the canonical B form are larger than those typically found for simulations of BDNA. For example, for the duplex d(CGCGAATTCGCG) the RMSd with respect to the canonical B form found in 10-20 ns trajectories in water (50), using the same force-field and simulation protocol, is around $2.3 \AA$ (1.9 $\AA$ if the crystal structure is used as reference), that is, around $1 \AA$ lower than the RMSds found here for oligonucleotides of the same length. As previously noted by experimentalists (see Introduction), this deviation suggests that polyd $(\mathrm{AT})_{\mathrm{n}}$ duplexes do not adopt a pure-canonical B structure in solution. However, for the sake of clarity we will continue denoting "B-form" those structures obtained in the $\mathrm{B}$ trajectories of polyd $(\mathrm{AT})_{\mathrm{n}}$ duplexes.

The hydrogen-bond (H-bond) patterns defining the Watson-Crick and Hoogsteen pairings are well preserved for $\mathrm{B}$ and apH trajectories (see Table 2). However, the total percentage of $\mathrm{H}$-bonds in the apH trajectories is typically larger than that found for the $\mathrm{B}$ ones. Very interestingly, the general stability of Watson-Crick and Hoogsteen H-bonds does not preclude the existence of reversible, partial breathing movements, like those shown in Figure 3. These movements occur in the nanosecond time scale and do not lead to a complete opening of the bases, which always retain at least one non-standard H-bond (see Figure 3). They are in general related to movements of thymine towards the major groove, and are more common in the B trajectories. 


\section{Description of the apH structure, interaction properties and dynamics.}

The analysis of the $5 \mathrm{~ns}$ trajectories of $\mathrm{d}(\mathrm{AT})_{5}$ and $\mathrm{d}(\mathrm{AT})_{6} \mathrm{apH}$ helices provides a complete structural picture of medium-sized apH helices in aqueous solution, complementing the X-ray information derived by Abrescia et al (17). The general shape of the apH helix is similar to that of a normal B-type duplex (see Figure 2), a quite surprising finding considering the dramatic differences in terms of nucleotide conformation and $\mathrm{H}$ bond recognition pattern between the two helices. The periodicity of the apH helix is similar to that of B-DNA (see Table 3 and ref. 51), the rise is only slightly larger (around $0.1 \AA$ ), the inclination is small and very similar to that found in B-trajectories, and the average phase angles suggest South and East-South puckerings, as usual in crystal structures of B-DNA (52). The minor groove in apH trajectories is around $2 \AA$ narrower than that found in B-like trajectories, while the major groove of the apH structures is around $3 \AA$ wider (see also Figure 2). There are differences between the two types of structures in the shortest $\mathrm{C} 1$ ' $-\mathrm{Cl}$ ' distances (around $2 \AA$ shorter for apH helices) and in the $\chi$ angles, which arise from the existence of the syn-2'deoxyadenosines in the apH helices (the average $\chi$ for apH 2'deoxyadenosines are around 50 degrees, in contrast to standard anti values of around -110 degrees). Finally, it is worth noting the similarity between the $\mathrm{X}$-ray structure of the apH helix and that found in MD simulations in solution (see Tables 1 and 3). In fact, the only noticeable discrepancy is found for the twist, which is 3 degrees smaller in MD simulations, reproducing a well known tendency of the AMBER force-field (see Table 3 and refs. $47 \mathrm{~b}$ and 53).

Despite the similar shape of $\mathrm{B}$ and apH helices in solution, it remains to be determined whether or not they exhibit similar interaction profiles. MIP maps (Figure 4) show that, as found for the B-DNA, the minor groove is the region with a stronger ability to interact with small cationic probes (smaller favourable regions are found in the major groove) in the apH structure. The shapes of MIP (isocontours of $-5 \mathrm{kcal} / \mathrm{mol}$ ) maps in apH and B helices are quite similar (see Figure 4). However, while the MIP isocontours are continuous for the B helix, they are not for the apH structures. This indicates that the minor 
groove in apH helices is slightly less charged than that of B helices (i.e slightly less polar). However, the differences found in the MIP maps are less relevant than those expected $a$ priori from the dramatic changes in the distribution of polar groups at the bottom of the grooves in B and apH helices (see Figure 1).

Both B and apH helices are very well solvated in our simulations. In average (for all the sequences and excluding the terminal base pairs), there are $26 \pm 7$ (B) and $27 \pm 12(\mathrm{apH})$ water molecules per step in direct contact (distance less than $3.5 \AA$ ) to any heteroatom of DNA, indicating a very similar hydration. For the B trajectories (Figure 4) small amounts of water are located in the major groove, and most of the regions of large water concentration $\left(>2.5 \mathrm{~g} / \mathrm{cm}^{3}\right)$ are in the minor groove, tracing clearly Dickerson's spine of hydration (52). For the apH structure the spine of hydration along the minor groove is also clearly defined, with waters located in the vicinities of O2 (thymines). Interestingly, a secondary spine of hydration (see details in Figure 5) is obtained along the major groove, with regions of intense hydration around N6 and N1 of adenines and O4 of thymines. A very dense hydration region is always located around N3 of adenines, bridging this atom to the phosphate group. The preferred regions of hydration found in MD simulation perfectly agree with highly ordered crystal waters located by Abrescia et al (17) in the major groove. Interestingly, the minor groove which is occupied by nucleobases in the crystal structure (17) is occupied by water in solution (see Figure 4). All these regions of large hydration correspond also to regions where waters with long residence times (up to $200 \mathrm{psec}$ ) are located (see Figure 5).

PCA provides a general view of the essential motions of the duplex in B and apH conformations. The first frequencies are very low (around 10-20 $\mathrm{cm}^{-1}$ ) and correspond to bending and untwisting movements for the two structures. In all cases, only very small differences are found between the frequencies of the first essential motions of $\mathrm{B}$ and apH structures. For example, the first three frequencies of the 10-mer sequence are (in $\mathrm{cm}^{-1}$ ) 15, 22 and 24 for the B helix, and 13, 16 and 27 for the apH structure. A quantitative comparison between the essential motions of $\mathrm{B}$ and apH helices by the absolute and relative 
similarity indexes $\gamma_{\mathrm{B} / \mathrm{pH}}$ and $\kappa_{\mathrm{B} / \mathrm{apH}}$ (eqs.2 and 3; Table 4) indicates a close similarity in the dynamics of the two helices.

In summary, MD simulations point out that many of the structural, reactive and dynamic properties of the apH helix are similar to those of the B helix, suggesting that proteins which evolved to recognize the general shape of B DNA could also recognize apH DNA. The subtle differences at the bottom of grooves might then alter the specificity of the interactions, opening a wide range of possible biological roles for the apH helix.

\section{Relative stability of $B$ and apH helices}

The preceding discussion points out that the crystal structure found by Abrescia et al. (17) is not a lattice-artifact, but an intrinsically stable structure of polyd(AT) ${ }_{n}$ in aqueous solution. However, to determine the possible biological role of apH helices, it is necessary to determine the relative stability of the apH helix versus the standard B-like conformation. For this purpose, we computed the free energy associated with each conformation using eq.1 (see Methods). It is worth noting that with 2-5 ns trajectories the average effective energy, $<\mathrm{E}_{\text {effec }}>=<\mathrm{E}_{\text {intra }}>+<\mathrm{G}_{\text {sol }}>$, can be estimated more accurately than the entropic term, which should need longer trajectories to converge (10a). Thus, we have performed two separate analysis: one quantitative for $<\mathrm{E}_{\text {effec }}>$ and another qualitative for $\Delta \mathrm{S}_{\text {intra. }}$.

The effective energies for the $\mathrm{B}$ and apH helices are shown in Table 5 (PB/SA values were used here to compute the solvation free energy; similar results were obtained with GB/SA method; see below). The similarity in the $\left\langle\mathrm{E}_{\text {effec }}>\right.$ values determined for the two conformations is evident. Thus, for the 10 and 12-mer oligonucleotides (those for which the longest trajectories are available) the $\left\langle\mathrm{E}_{\text {effec }}>\right.$ values differ by only 0 and 3 $\mathrm{kcal} / \mathrm{mol}$, i.e., less than $0.06 \%$ the total energy value, and lie within the estimated error of the averages (see Table 5).

A more quantitative estimate of the relative effective energy for B and apH helices can be obtained (10a) taking advantage of the linear dependence between $\left\langle\mathrm{E}_{\text {effec }}>\right.$ and the 
length (n) of the oligonucleotide $\left(\mathrm{r}^{2}=1.0000\right.$ for the regression line $\left\langle E_{\text {effect }}\right\rangle=a \times(n-2)+b$ in all cases). Typical errors (see Figure 6) in the slope ( $a$ : helix-growth stabilization factor) and intercept ( $b$ : nucleation energy) are only around $0.2-0.5$ and $2-4 \mathrm{kcal} / \mathrm{mol}$, respectively. The regression equations (see Figure 6) suggest that there is not significant preference for either $\mathrm{B}$ or apH conformations in aqueous solution in terms of $\left\langle\mathrm{E}_{\text {effec }}\right\rangle$ (the same trends were found when solvation was determined using the GB/SA model, and when PB/SA calculations were repeated considering the internal dielectric constant equal to 2; data not shown). This supports the equivalence in effective energy between B and apH structures, and suggests that the population of the two helices in solution might depend on the existence of cofactors, specific hydration waters, and/or entropic considerations. Due to the similar degree of hydration in the first hydration shell (see above) no large differences in stability are expected arising from interaction with specific water molecules. The impact of entropic effects and cofactors will be discussed below.

To gain further insight into the similar effective energies of $\mathrm{B}$ and apH structures, we examined their intramolecular and solvation contributions (see eq.1 and Table 5). As found in previous works (10a), there is a second-order polynomial relationship between the size of the duplex and its solvation and intramolecular energy components. Then, the linear relationship found for $\mathrm{E}_{\text {effec }}$ stems from the cancellation of the second-order coefficients for the solvation and intramolecular terms (see Figure 7). In general, for medium to long oligonucleotides the B form is slightly favoured by solvation and slightly disfavoured by intramolecular interactions with respect to the apH conformation. However, the differences are small (see Table 5 and Figure 7), and we can conclude that not only the B and apH structures are similar in terms of the effective energies, but also in their solvation and intramolecular contributions.

The similar solvation free energy of apH and B helices is not surprising considering the solvation maps (Figures 4 and 5) and the number of water molecules in the first hydration shell (see above). However, the similar intramolecular energy is surprising considering that apH duplexes imply the existence of syn-adenosines, which are expected to be less stable than the anti conformation. However, NMR data in DMSO and water (54) 
show that the free energy difference between syn and anti conformations is small (0.4-0.6 $\mathrm{kcal} / \mathrm{mol}$ for adenosine). A similar value $(0.7 \mathrm{kcal} / \mathrm{mol})$ is also found by restricted AMBER99 optimizations of syn and anti adenosines at the MD-averaged $\chi$ torsions found in our B and apH simulations. This small energy penalty and others related to unfavourable backbone-backbone interactions in the apH helix are compensated by the better stacking and H-bond interactions in Hoogsteen A-T pairs. Thus, AMBER calculations in the gas phase using the MD-averaged structures of the Watson-Crick and Hoogsteen pairs show interaction energies of -12.7 (WC) and $-13.2(\mathrm{H}) \mathrm{kcal} / \mathrm{mol}$, i.e. the Hoogsteen H-bond pattern is around $0.6 \mathrm{kcal} / \mathrm{mol}$ more stable than the Watson-Crick scheme (55). Linear regression lines (see Figure 8) between the H-bonding energy and the length of the oligonucleotides suggest that, in the DNA environment, the Hoogsteen scheme is around $1.3 \mathrm{kcal} / \mathrm{mol} \times$ step more stable than the Watson-Crick pattern. Similarly (Figure 8) the stacking energy of the Hoogsteen pair is on average around $1.9 \mathrm{kcal} / \mathrm{mol} \mathrm{x}$ step more stable than the Watson-Crick one in the DNA environment.

In summary, the MM/PB-SA (and MM/GB-SA) analysis, despite their shortcomings, strongly suggest similar stability for the $\mathrm{B}$ and apH structures in aqueous solution in terms of intramolecular energy and solvation free energy. Then, the relative population of the two helices might be determined by entropic considerations. The structural and dynamical analysis described above points out that the $\mathrm{B}$ form is more flexible than the apH one, the former being then entropically favoured. To obtain more detailed information, entropic calculations were performed using Schlitter's method, as described in Methods. Though accurate entropic calculations would need longer trajectories than those performed here, the analysis of 2-5 ns trajectories can still be useful to obtain relative entropy values.

Entropy calculations were then performed for all the trajectories in the 1-2 ns period finding linear relationships with the length of the oligonucleotides $\left(r^{2}>0.993\right.$; see Figure 9$)$. The regression lines confirm that the B form is entropically favoured compared to the apH helix in all cases, and that the difference slightly increases with the length of the oligonucleotide. For example, the regression equations indicate that for the 10 and 12-mer 
oligonucleotides the B structure is favoured by 0.11 and $0.14 \mathrm{kcal} / \mathrm{molK}$ with respect to the $\mathrm{apH}$ helix. Similar values are obtained when entropies are calculated using the $5 \mathrm{~ns}$ trajectories and extrapolated to infinite simulation time using Harris's method (43): 0.15 (10-mer) and 0.21 (12-mer) $\mathrm{kcal} / \mathrm{molK}$. These values suggest that at $\mathrm{T}=298 \mathrm{~K}$ the entropic effect will be around 45 (10 mer) 63 (12-mer) $\mathrm{kcal} / \mathrm{mol}$, a sizeable difference considering the small differences in effective energies, which would lead to a predominance of the $\mathrm{B}$ form in aqueous solution. We can expect that when the flexibility of the DNA is restricted by the presence of drugs, proteins, or lattice constraints, the intrinsic flexibility of the DNA is drastically altered, modifying then the entropy balance between B and apH helices. In the latter cases, the apH helix could become competitive with the B structure, explaining the existence of apH structures in the crystal (17), and the presence of apH recognition modes in complexes of DNA with either intercalators or proteins (see Introduction).

\section{Chimeras of B and apH helices}

In long DNA fragments, the apH structure may occur in short fragments surrounded by the B-structure, and often the fragments will contain both $\mathrm{d}(\mathrm{AT})$ and $\mathrm{d}(\mathrm{AA})$ steps. Accordingly, whether or not an apH track can be inserted into a B canonical structure without inducing large distortions, and whether or not it can exist in polyd(A) sequences are key issues to guess the biological importance of apH helices. Test calculations of poly $(\mathrm{dA})_{10}$ (data not shown, but available upon request) show that the apH helix can also be stable: the average effective energy (eq. 1) found in 2 ns trajectories of the apH helix ($2663 \mathrm{kcal} / \mathrm{mol}$ ) was almost identical to that found for a WC duplex of the same sequence ($2662 \mathrm{kcal} / \mathrm{mol}$; reference 10a), and slightly more negative than that found for parallel stranded duplexes with reverse Watson-Crick (-2642 kcal/mol; 10a) and Hoogsteen pairings (-2657 $\mathrm{kcal} / \mathrm{mol}$; 10a). In summary, our MD simulations suggest that the apH helix can exist also in poly $(\mathrm{dA})$ sequences, opening then the window of structures for which apH helices are possible. 
The impact of having apH sequences inserted in B-DNA was studied in two chimeric 11-mer sequences: d(ATATAAAATAT) and d(ATATAAAATAT), where the steps having Watson-Crick recognition mode are in plain, and those with the apH pattern in bold. Unrestricted NPT trajectories collected for 2 ns after equilibration are very stable, and reveal no strong destabilization, conformational transition or unfolding (see Figures 10 and 11). The backbone RMSd with respect to the average structures are around $1.3 \AA$ in the two cases (Figure 11), thus confirming the stability of the trajectories. The apH rich d(ATATAAAATAT) structure is closer to an apH helix than to a $\mathrm{B}$ conformation (backbone RMSd of 1.9 and $2.4 \AA$, respectively), while the Watson-Crick rich d(ATATAAAATAT) structure is intermediate between apH and B conformations (backbone RMSd of 1.9 and $1.8 \AA$, respectively). Very interestingly, the chimeras maintain clearly separable conformations corresponding to the regions with apH or Watson-Crick pairings (see Figure 10). Such a structural difference has a clear impact in the recognition properties of the helix (see Figure 10). Thus, for the d(ATATAAAATAT) helix, the -5 $\mathrm{kcal} / \mathrm{mol}$ contour is discontinuous at the central region, as expected for an apH helix, and contiguous in the rest, as in a canonical B-helix (see Figure 4). On the contrary, the reverse trend occurs for the d(ATATAAAATAT) sequence. This finding indicates that not only $\mathrm{apH}$ and $\mathrm{B}$ helices are compatible in the same oligonucleotide, but that even when they are contiguous each helix fragment preserves its own structural and reactive integrity.

\section{CONCLUSIONS}

In summary, the preceding results show that the apH structure is a thermodynamic stable conformation, which might compete with the B conformation if the flexibility of the helix is altered. However, for the sequences studied MD simulations favour the B form in dilute aqueous solution of lineal DNAs in the absence of cofactors. Interestingly, the structural, dynamical and reactive properties of the apH helices are not extremely different to those of the B helix. However, the small changes should allow proteins and drugs to distinguish between these two helical models. MD simulations suggest that apH structures can coexist next to B-type structures, demonstrating again the extreme plasticity of DNA. If 
kinetic factors are not limiting (the $s y n \leftarrow \rightarrow$ anti barrier is around $6.2 \mathrm{kcal} / \mathrm{mol}$; 56), AT rich regions might adopt $\mathrm{B}$ and apH forms, and this possibility might be exploited to tune the interaction with specific proteins.

\section{ACKNOWLEDGMENTS}

We are indebted to X.-J.Lu and W. Olson and for a copy of X3DNA and help in the use of the code for a non standard structure. We also thank D.Bashford for a copy of his MEAD program. The Centre de Supercomputació de Catalunya (CESCA) and the Spanish Ministry of Science and Technology (SAF2002-4282, PM99-0046 and BIO2003-06848) are acknowledged for financial support. 


\section{REFERENCES}

(1) Watson,J.D.; Crick.F.H.C. Nature, 1953, 171, 737.

(2) (a) Saenger,W. Principles of Nucleic Acid Structure. Springer-Verlag. New York. 1984.(b) Ghosh,A.; Manju,B. Acta Cryst., 2003, D59, 620.

(3) Bloomfield,v.A.; Crothers,D.N.; Tinoco,I. (eds). Nucleic Acids: Structures, Properties and Functions. University Scince Books. Sausalito 2000.

(4) Blackburn,G.M.; Gait,M.J. (eds). Nucleic Acids in Chemistry and Biology. IRL Press. Oxford. 1990.

(5) Wang,A.H.-J.; Quiqkey,G.J.; Kolpak,F.J.; Crawford,J.L.; van Boom,J.H.; van der Marel,G.; Rich,A. Nature, 1979, 282, 680.

(6) Laughlan,G.; Murchie,A.I.H.; Norman,D.G.; Moore,M.H.; Moody,P.C.E.; Lilley,D.M.; Luisi,B. Science, 1994, 265, 520.

(7) Robles,J.; Grandas,A.; Pedroso,E.; Luque,F.J.; Eritja,R.; Orozco,M. Current Org. Chem., 2002, 6, 1333; and references therein.

(8) Gehring,K.; Leroy,J.L.; Guéron,M. Nature, 1993, 363, 561.

(9) Gray,D.M.; Vaughn,M.; Ratliff,R.L.; Hayes,F.N. Nucleic Acids Res., 1980, 8, 3695.

(10) (a) Cubero,E.; Luque,F.J.; Orozco,M. J.Am.Chem.Soc., 2001, 123, 12018. (b) Cubero,E.; Aviñó,A.; de la Torre,B.G., Frieden,M; Eritja,R.; Luque,F.J.; González,C.; Orozco,M. J.Am.Chem.Soc., 2002 124, 3133.

(11) Viswamitra,M.A.; Kennard,P.; Jones,P.G.; Sheldrick,G.M.; Salisbury,S.; Falvello,L.; Shakked,Z. Nature, 1978, 273, 687.

(12) Viswamitra,M.A.; Shakked,Z.; Sheldrick,J.G.M.; Salisbury,S.A.; Kennard,O. Biopolymers, 1982, 21, 513.

(13) Guzikevich-Guerstein,G.; Shakked,Z. Nat. Struct. Biol., 1996, 3, 32.

(14) Radwan,M.M.; Wilson,H.R. Int.J.Biol.Macromol., 1982, 4, 145.

(15) Davis,D.R.; Baldwin,R.L. J.Mol.Biol., 1963, 6, 251.

(16) Brahms,S.; Brahms,J.; Van Holde,K.E. Proc. Natl. Acad. Sci. USA, 1976, 73, 3453.

(17) (a) Abrescia,N.G.A.; Thompson,A.; Dinh,T.H.; Subirana,J.A. Proc.Natl.Acad.Sci.USA, 2002 99, 2806; (b) Abrescia,N.G.A.; Subirana,J.A. Acta Cryst., 2002, D58, 2205. 
(18) Aishima,J.; Gitti,R.K.; Noah,J.E.; Gan,H.H.; Schlick,T.; Wolberger,C. Nucleic Acids Res., 2002, 30, 5244.

(19) Lefèvre,J.F.; Lane,A.N.; Jardetzky,O. Biochemistry, 1988, 27, 1086.

(20) Schmitz,U.; Sethson,I.; Egan,W.M.; James,T.L. J.Mol.Biol., 1992, 227, 510.

(21) McAtter,K.; Ellis,P.D.; Kennedy,M.A. Nucleic Acids Res., 1995, 23, 3962.

(22) Hoogsteen,K. Acta Crystallogr., 1959, 12, 822.

(23) Sponer,J.; Leszczynski,J.; Hobza,P. J.Phys.Chem., 1996, 100, 1969.

(24) Shields,G.C.; Laughton,C.A.; Orozco,M. J.Am.Chem.Soc., 1997, 119, 7563.

(25) (a) Voet,D.; Rich,A. Prog. Nucl. Acid. Res. Mol. Biol., 1970, 10, 183. (b) Leontis,N.B.; Westhof,E. Quart. Rev. Biophys. 1998, 31, 399. (c) Nolten,G.M.J.; Sijtema,N.M.; Otto,C. Biochemistry, 1997, 36, 13241.

(26) (a) Pauling,L.; Corey,R.B. Proc. Natl. Acad. Sci. USA, 1953, 39, 84. (b) Felsenfeld,G., Davis,D.R.; Rich,A. J.Am.Chem.Soc., 1957, 79, 2023.

(27) Patikoglou,G.A.; Kim,J.L.; Sun,L.; Yang,S.-H.; Kodadek,T.; Burley,S.K. Genes \& Development, 1999, 13, 3217.

(28) Rice,P.A.; Yang,S.; Mizuuchi,K.; Nash,H.A. Cell, 1996, 87, 1295.

(29) (a) Wang,A.H.; Ughetto,G.; Quigley,G.J.; Hakoshima,T.; van der Marel,G.A.; van Boom,J.H.; Rich,A. Science, 1984, 225, 1115. (b) Gilbert,D.E.; van der Marel,G.A.; van Boom,J.H.; Feigon,J. Proc. Natl. Acad. Sci. USA, 1989, 86, 3006.

(30) (a) Moreau,J.; Maschat,M.F.; Kejzlarova-Lepesant,J.; Lepesant,J.-A.; Scherrer,K. Nature, 1982, 295, 260. (b) Oosumi,T.; Garlick,B.; Belknap,W.R. Proc. Natl. Acad. Sci. USA., 1995, 92, 8886.

(31) Arnott,S.; Hukins,D.W.L. Biochem.Biophys.Res.Commun. 1972, 47, 1504.

(32) (a) Soliva,R.; Laughton,C.A.; Luque,F.J.; Orozco,M. J.Am.Chem.Soc., 1998, 120, 11226. (b) Shields,G.C.; Laughton,C.A.; Orozco,M. J.Am.Chem.Soc., 1998, 120, 5895.

(33) Darden,T.A.; D.M.York,D.M.; Pedersen,L.G. J.Chem.Phys., 1993, 98, 10089.

(34) Ryckaert, J.P., Ciccotti, G., Berendsen, H.J.C. J. Comput. Phys., 1977, 23, 327.

(a) Cornell,W.D.; Cieplak,P.; Bayly,C.I.; Gould,I.R.; Merz,K.M.; Fergurson,D.M.; Spellmeyer,D.C.; Fox,T.; Caldwell,J.W.; Kollman,P.A. J.Am.Chem.Soc., 1995, 117, 
5179. (b) Cheatham,T.E.; Cieplak,P.; Kollman,P.A. J.Biomol.Struct.Dyn., 1999, 16, 845.

(36) Jorgensen,W.L. ; Chandrasekhar,J. ; Madura,J.D. ; Impey,R.W. ; Klein,M.L. J.Chem.Phys., 1983, 79, 926.

(37) Case, D. A.; Pearlman, D. A.; Caldwell, J. W.; Cheatham, T. E., III; Ross, W. S.; Simmerling, C. L.; Darden, T. L.; Marz, K. M.; Stanton, R. V.; Cheng, A. L.; Vincent, J. J.; Crowley, M.; Tsui, V.; Radmer, R. J.; Duan, Y.; Pitera, J.; Massova, I.; Seibel, G. L.; Singh, U. C.; Weiner, P. K.; Kollman, P. A. AMBER6. University of California. San Francisco. 1999.

(38) Kollman,P.A.; Massova,I.; Reyes,C.; Kuhn,B.; Huo,S.; Chong,L.; Lee,M.; Lee,T.; Duan,Y.; Wang,W.; Donini,O.; Cieplak,P.; Srinivasan,J.; Case,D.; Cheatham,T.E. Acc.Chem.Res., 2000, 33, 889.

(39) Orozco,M.; Luque,F.J. Chem.Rev., 2000, 100, 4187.

(40) (a) Bashford,D.; Gerwert,K. J.Mol.Biol., 1992, 224, 473. (b) Bashford,D. In Scientific Computing in Object-Oriented parallel environments; Reynders,J.V.M., Tholburn,M., Eds.; Springer: Berlin, 1997, pp 233-240.

(41) Still,W.C.; Tempcyck,A.; Hawley, R.C.; Hendrickson.T. J.Am.Chem.Soc., 1990, $112,6127$.

(42) Schliter,J. Chem.Phys.Let., 1993, 215, 617.

(43) Harris,S.; Gavathiotis,E.; Searle,M.S.; Orozco,M.; Laughton,C.A J.Am.Chem.Soc., 2001, 123, 12658 .

(44) Andricioaei,I.; Karplus,M. J.Chem.Phys., 2001, 115, 6289.

(45) Gelpí,J.L.; Kalko,S.; de la Cruz,X.; Barril,X.; Cirera,J.; Luque,F.J.; Orozco,M. Proteins, 2001, 45, 428.

(46) Sherer,E.; Harris,S.A.; Soliva,R.; Orozco,M.; Laughton,C.A. J.Am.Chem.Soc., 1999, 121, 5981.

(47) (a) Rueda,M.; Kalko,S.G.; Luque,F.J.; Orozco,M. J.Am.Chem.Soc., 2003, 125, 8007. (b) Orozco,M.; Noy,A.; Pérez,A.; Luque,F.J. Chem.Soc.Rev. 2003, 32.

(48) Lu,X,-J.; Olson,W.K. X3DNA Program. Rutgers University. 2001. 
(49) Lavery,R.; Sklenar,J. J.Biomol.Struct.Dyn., 1988, 6, 63.

(50) Rueda,M.; Cubero,E.; Laughton,C.A.; Orozco,M. To be published.

(51) The existence of syn adenines makes very difficult the helical analysis of apH helices, even when the very flexible X3DNA program is used. The only parameters that can be safely derived from helical analysis are those displayed in Table 3.

(52) Drew, H.R.; Wing,R.M.; Tanako,T., Broka,C., Tanaka,S., Itakura,K., Dickerson,R. Proc. Natl. Acad. Sci. USA, 1981, 78, 2179.

(53) Cheatham,T.E.; Young,M.A. Biopolymers, 2001, 56, 232.

(54) Stolarski,R.; Pohorille,A.; Dudycz,L.; Shugar,D. Biochim.Biophys.Acta, 1980, 610, 1.

(55) These values agree very well with our own B3LYP/6-31G(d) estimates (-12.1 and -12.6 $\mathrm{kcal} / \mathrm{mol}$ respectively), and with MP2/6-31G*(0.25) estimates by Sponer and Hobza (11.8 (WC) and $-12.7 \mathrm{kcal} / \mathrm{mol}$ respectively from reference 23 ).

(56) Rhodes,L.M.; Schimmel,P.R. Biochemistry, 1971, 10, 4426. 
(A)

\begin{tabular}{|c|c|c|c|}
\hline Structure & Average $^{\#}$ & B-DNA $^{\text {\% }}$ & A-DNA \\
\hline B4 & $1.0 \pm 0.2$ & $1.4 \pm 0.2$ & $1.9 \pm 0.2$ \\
\hline B6 & $1.1 \pm 0.2$ & $2.1 \pm 0.3$ & $2.3 \pm 0.2$ \\
\hline B8 & $1.4 \pm 0.3$ & $2.5 \pm 0.5$ & $3.4 \pm 0.6$ \\
\hline B10* & $1.5 \pm 0.2$ & $2.9 \pm 0.5$ & $3.7 \pm 0.5$ \\
\hline B12* & $1.7 \pm 0.4$ & $3.3 \pm 0.7$ & $4.4 \pm 0.7$ \\
\hline B14 & $1.7 \pm 0.3$ & $3.4 \pm 0.6$ & $5.0 \pm 0.6$ \\
\hline B16 & $2.1 \pm 0.6$ & $3.9 \pm 0.9$ & $5.0 \pm 0.8$ \\
\hline
\end{tabular}

(B)

\begin{tabular}{|c|c|c|}
\hline Structure & Average $^{\#}$ & Crystal structure $^{\text {\& }}$ \\
\hline $\mathrm{apH} 4$ & $1.5 \pm 0.6$ & $1.5 \pm 0.2$ \\
\hline $\mathrm{apH6}$ & $1.0 \pm 0.2$ & $1.0 \pm 0.2$ \\
\hline $\mathrm{apH} 8$ & $0.8 \pm 0.2$ & $1.1 \pm 0.2$ \\
\hline $\mathrm{apH10}$ & $1.3 \pm 0.3$ & $1.9 \pm 0.5$ \\
\hline $\mathrm{apH} 12^{*}$ & $1.3 \pm 0.3$ & $1.5 \pm 0.3$ \\
\hline $\mathrm{apH14}$ & $1.5 \pm 0.4$ & $1.5 \pm 0.3$ \\
\hline $\mathrm{apH} 16$ & $1.3 \pm 0.3$ & $1.6 \pm 0.3$ \\
\hline
\end{tabular}

Table 1. All-atom root mean square deviations (RMSd; $⿱$ Á) of B-DNA (top) and apH-DNA (bottom) trajectories with respect to different reference structures. Standard deviations (SD) in the averages are also displayed.

* 5 ns trajectories, the rest are only 2 ns long.

${ }^{\#}$ MD-averaged structures obtained using the last 1 or 4 ns of trajectories.

${ }^{\%}$ Arnott's structures (see reference 31 ).

${ }^{\&}$ Structures built by joining two 5-mer structures in PDB entry 1gpu.pdb followed by restricted minimization. 


\begin{tabular}{|c|c||c|c|}
\hline Structure & WC H-bonds & Structure & H H-bonds \\
\hline B4 & 96.6 & apH4 & 73.8 \\
\hline B6 & 70.4 & apH6 & 97.3 \\
\hline B8 & 88.6 & apH8 & 97.2 \\
\hline B10 & 94.4 & apH10 & 91.9 \\
\hline B12 & 91.8 & apH12 & 94.8 \\
\hline B14 & 92.7 & apH14 & 96.5 \\
\hline B16 & 93.3 & apH16 & 97.1 \\
\hline
\end{tabular}

Table 2. Occurrence (in \% with respect to the maximum number of hydrogen-bond interactions) of canonical Watson-Crick (WC) and Hoogsteen (H) hydrogen bonds for the different trajectories. 


\begin{tabular}{|c|c|c|c|c|c|c|}
\hline Parameter & B10 & B12 & apH10 & apH12 & B-DNA & X-ray \\
\hline $\mathrm{C} 1^{\prime}-\mathrm{C} 1^{\prime \&}$ & 10.4 & 10.3 & 7.9 & 8.3 & 10.9 & 8.2 \\
\hline Inclination & 4.5 & 6.4 & 3.2 & 3.4 & -5.0 & 2.2 \\
\hline Helical twist & 33.2 & 32.3 & 32.0 & 32.0 & 36.0 & 35.3 \\
\hline Helical rise & 3.1 & 3.1 & 3.3 & 3.2 & 3.4 & 3.3 \\
\hline Phase & 132.0 & 128.4 & 118.9 & 121.1 & 191.7 & 155.1 \\
\hline Amplitude & 36.5 & 35.3 & 42.3 & 40.9 & 35.7 & 35.6 \\
\hline m-groove ${ }^{\#}$ & 12.9 & 13.0 & 10.6 & 10.4 & 11.7 & $9.3-11$ \\
\hline M-groove & 18.7 & 18.8 & 21.9 & 21.6 & 16.9 & $\approx 20$ \\
\hline
\end{tabular}

Table 3. Key helical parameters for the MD-averaged B and apH helices (for 10 and 12mer oligonucleotides). The helical parameters of a standard B-DNA and of the structure generated from crystal data in ref. 17 are also displayed. Distances are in $\AA$ and angles in degrees.

${ }^{\&}$ Minimum C1'-C1' distance.

${ }^{\$}$ Crystal structure from 1gpu.pdb (see text).

${ }^{\text {\# }}$ Determined from the shortest P-P distances across the groove 


\section{(10 mer)}

\begin{tabular}{|c|c|c|}
\cline { 2 - 3 } \multicolumn{1}{c|}{} & B & apH \\
\hline B & 0.7 & 0.5 \\
\hline $\mathbf{a p H}$ & & 0.8 \\
\hline
\end{tabular}

(12 mer)

\begin{tabular}{|c|c|c|}
\cline { 2 - 3 } \multicolumn{1}{c|}{} & B & apH \\
\hline B & 0.7 & 0.6 \\
\hline apH & & 0.7 \\
\hline
\end{tabular}

Table 4. Similarity indexes $\gamma$ obtained using eq. 1 and the 10 first principal components for the 10 and 12-mer duplexes in B and apH structures. Similarity indexes $\gamma_{\mathrm{B} / \mathrm{apH}}$ were computed using the last $4 \mathrm{~ns}$ of both trajectories. Self-similarity indexes $\gamma_{\mathrm{B} / \mathrm{B}}$ and $\gamma_{\mathrm{apH} / \mathrm{apH}}$ were determined comparing the first $(1 \rightarrow 3 \mathrm{~ns})$ and second $(3 \rightarrow 5 \mathrm{~ns})$ halves of the trajectories. 


\begin{tabular}{|c|c|c|}
\hline Length-2 & B & apH \\
\hline 2 & $\mathbf{- 6 3 7 . 5} \pm \mathbf{0 . 4}$ & $\mathbf{- 6 3 5 . 4} \pm \mathbf{0 . 4}$ \\
& $-544.0 \pm 0.4 /-93.5 \pm 0.2$ & $-559.9 \pm 0.4 /-75.6 \pm 0.2$ \\
\hline 4 & $\mathbf{- 1 3 6 3 . 0} \pm \mathbf{0 . 7}$ & $\mathbf{- 1 3 6 4 . 9} \pm \mathbf{0 . 6}$ \\
& $-1461.3 \pm 0.6 / 98.2 \pm 0.3$ & $-1484.3 \pm 0.5 / 119.4 \pm 0.2$ \\
\hline 6 & $\mathbf{- 2 0 9 3 . 3} \pm \mathbf{1 . 6}$ & $\mathbf{- 2 0 9 5 . 2} \pm \mathbf{1 . 1}$ \\
\hline 8 & $-2647.0 \pm 1.5 / 553.7 \pm 0.5$ & $-2682.3 \pm 1.0 / 587.1 \pm 0.4$ \\
\hline 10 & $\mathbf{- 2 8 1 9 . 7} \pm \mathbf{1 . 9}$ & $\mathbf{- 2 8 2 1 . 9} \pm \mathbf{1 . 9}$ \\
\hline & $-4075.7 \pm 1.8 / 1256.1 \pm 0.6$ & $-4083.8 \pm 1.9 / 1261.9 \pm 0.6$ \\
\hline 12 & $\mathbf{- 3 5 4 8 . 9} \pm \mathbf{3 . 0}$ & $\mathbf{- 3 5 4 8 . 9} \pm \mathbf{2 . 6}$ \\
\hline & $-5681.4 \pm 2.9 / 2132.5 \pm 1.0$ & $-5681.5 \pm 2.4 / 2132.6 \pm 0.8$ \\
\hline 14 & $\mathbf{- 4 2 6 9 . 9} \pm \mathbf{3 . 3}$ & $\mathbf{- 4 2 7 6 . 5} \pm \mathbf{3 . 4}$ \\
& $-7453.7 \pm 3.2 / 3183.8 \pm 1.0$ & $-7387.8 \pm 4.1 / 3111.3 \pm 1.3$ \\
\hline & $\mathbf{- 4 9 9 9 . 7} \pm \mathbf{4 . 7}$ & $\mathbf{- 5 0 0 1 . 5} \pm \mathbf{3 . 6}$ \\
& $-9350.3 \pm 4.4 / 4350.6 \pm 1.4$ & $-9297.1 \pm 3.4 / 4295.6 \pm 1.1$ \\
\hline
\end{tabular}

Table 5. Effective energy $\left(<\mathrm{E}_{\mathrm{effec}}>\right.$ in bold), solvation and their intramolecular and solvation contributions $\left(<\mathrm{G}_{\mathrm{sol}}>\right.$ in roman $/<\mathrm{E}_{\mathrm{intra}}>$ in italics $)$ computed for the different oligonucleotides in $\mathrm{B}$ and apH conformations. Values determined using PB/SA estimates of solvation free energy for internal and external dielectric constants of 1 and 80 . Values and their standard errors (in parenthesis) are in $\mathrm{kcal} / \mathrm{mol}$. 
Cubero et al., J.Am.Chem.Soc. 2003

\section{LEGENDS TO FIGURES}

Figure 1. Schematic representation of the possible recognition patterns between neutral adenine and thymine.

Figure 2. Representation of the MD-averaged structure of the $d(A T)_{n / 2}$ for $n=10$ in the $B$ (left) and antiparallel Hoogsteen conformations (right). See text for details.

Figure 3. Evolution of key H-bond distances (in Å) in trajectories B (10-mer) and apH (12mer) trajectories. The existence of partial breathing movements is seen in a temporary lost of canonical Watson-Crick or Hoogsteen hydrogen bonds, and the simultaneous gain of one additional non-canonical hydrogen bond. Colour code is as follow. TOP: black: H6 $\left(\mathrm{A}_{7}\right)-$ O4( $\left.\mathrm{T}_{14}\right)$; red: $\mathrm{N} 1\left(\mathrm{~A}_{7}\right)-\mathrm{H} 3\left(\mathrm{~T}_{14}\right)$; green $\mathrm{H} 2\left(\mathrm{~A}_{7}\right)-\mathrm{O} 2\left(\mathrm{~T}_{14}\right)$ and blue H6( $\left.\mathrm{A}_{7}\right)-\mathrm{O} 2\left(\mathrm{~T}_{14}\right)$. BOTTOM: black: $\mathrm{O} 4\left(\mathrm{~T}_{6}\right)-\mathrm{H} 6\left(\mathrm{~A}_{19}\right)$; red: $\mathrm{H} 3\left(\mathrm{~T}_{6}\right)-\mathrm{N} 7\left(\mathrm{~A}_{19}\right)$; green $\mathrm{O} 2\left(\mathrm{~T}_{6}\right)-\mathrm{H} 8\left(\mathrm{~A}_{19}\right)$ and blue $\mathrm{O} 2\left(\mathrm{~T}_{6}\right)$ H6( $\left.A_{19}\right)$.

Figure 4. Molecular Interaction Potentials contours $(-5 \mathrm{kcal} / \mathrm{mol}$; TOP) and solvation maps $(2.5 \mathrm{~g} / \mathrm{ml}$; BOTTOM) for the B (right) and apH (left) helices. The data for the 10 mer oligonucleotides are shown in all cases (similar profiles are obtained for the other sequences).

Figure 5. Details of regions of specific hydration in the apH helix ( $2.5 \mathrm{~g} / \mathrm{ml}$ contours) along the major groove. Data correspond to the 10-mer structure. Water molecules with long residence time are located in the areas. Residence times for waters bound to $\mathrm{O}_{2}$ and $\mathrm{O}_{4}$ are very large (150-200 ps), waters bound to $\mathrm{N}_{3}$ show also large residence times (around 50$100 \mathrm{ps}$ ), water around $\mathrm{N}_{1}$ show residence times of 30-50 ps, and those around $\mathrm{N}_{6}$ have residence times around $20 \mathrm{psec}$.

Figure 6. Representation of the variation of the effective energy $<\mathrm{E}_{\text {effec }}>=<\mathrm{E}_{\text {intra }}>+<\mathrm{G}_{\text {sol }}>$ as a function of the length of the oligonucleotide for $\mathrm{B}$ and apH conformations. TOP: Solvation computed using the GB/SA method. BOTTOM: Solvation computed using the 
$\mathrm{PB} / \mathrm{SA}$ approach. In both cases interior and exterior dielectric constants are 1 and 80. All values are in $\mathrm{kcal} / \mathrm{mol}$.

Figure 7. Representation of the variation of the solvation free energy (TOP: $<\mathrm{G}_{\mathrm{sol}}>$ ) and intramolecular energy (BOTTOM: $\mathrm{E}_{\mathrm{intra}}>$ ) as a function of the length of the oligonucleotides for $\mathrm{B}$ and $\mathrm{apH}$ conformations (all values are in $\mathrm{kca} / \mathrm{mol}$ ). Solvation values displayed here correspond to the $\mathrm{PB} / \mathrm{SA}$ calculations with dielectric constants of 1 (interior) and 80 (exterior). Identical profiles where obtained using other solvation models (see text and Table 5). Parabolic fits correspond to the equation: $y=a_{2} x^{2}+a_{1} x+b$

Figure 8. Representation of the variation of the hydrogen bond (TOP) and stacking energies (BOTTOM) as a function of the length of the oligonucleotides for B and apH conformations (all values are in $\mathrm{kcal} / \mathrm{mol}$ ).

Figure 9. Representation of the variation of the entropy (in $\mathrm{kcal} / \mathrm{mol} \mathrm{K}$ ) as a function of the length of the oligonucleotides for B and apH conformations (all values are in $\mathrm{kcal} / \mathrm{mol}$ ). All values were obtained from $1 \mathrm{~ns}$ samplings (see text).

Figure 10. MIP (-5 kcal $/ \mathrm{mol})$ contour for the d(ATATAAAATAT) (LEFT) and d(ATATAAAATAT) structures. The apH portions are colored in green and the WC portions are in blue. Bold means Hoogsteen pairings.

Figure 11. Backbone root mean square deviations (RMSd in $\AA$ ) of the d(ATATAAAATAT) (TOP) and d(ATATAAAATAT) trajectories with respect to: RED: the MD-averaged structures; GREEN: the B- structure; and BLUE: the apH structure. Averaged RMSd with respect to the average, B and apH structures are 1.24, 2.44 and 1.91 $\AA$ for the d(ATATAAAATAT) structure, and $1.37, \quad 1.91$ and $1.80 \AA$ for the d(ATATAAAATAT) structure. Bold means Hoogsteen pairings. 
Cubero et al., J.Am.Chem.Soc. 2003 